\section{Korrespondenzadresse}

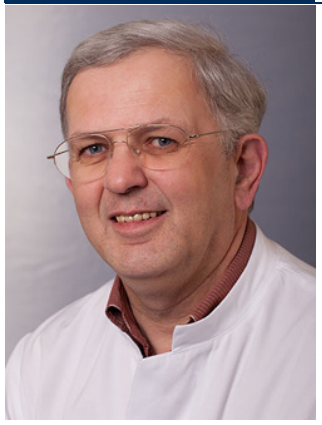

Univ.-Prof. Dr. H.-J. Trappe

Medizinische Universitätsklinik II (Schwerpunkte Kardiologie und Angiologie), RuhrUniversität Bochum Hölkeskampring 40, 44625 Herne, Deutschland hans-joachim.trappe@ruhr-uni-bochum.de

\section{Einhaltung ethischer Richtlinien}

Interessenkonflikt. H.-J. Trappe gibt an, dass kein Interessenkonflikt besteht.

Für diesen Beitrag wurden von den Autoren keine Studien an Menschen oder Tieren durchgeführt. Für die aufgeführten Studien gelten die jeweils dort angegebenen ethischen Richtlinien.

\section{Literatur}

1. Trappe HJ, Schuster HP (Hrsg) (2020) EKG-Kurs für Isabel, 8. Aufl. Thieme, Stuttgart

Kardiologe 2021 · 15:654

https://doi.org/10.1007/s12181-021-00516-2

Online publiziert: 2. November 2021

๑) Deutsche Gesellschaft für Kardiologie - Herz- und Kreislaufforschung e.V. Published by Springer Medizin Verlag GmbH, ein Teil von Springer Nature - all rights reserved 2021

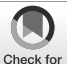

\title{
Erratum zu: Hypertriglyzeridämie verstehen
}

\section{Was ist zu tun? Ist etwas zu tun?}

\author{
Theresa Berent ${ }^{1,2} \cdot$ Kurt Derfler $^{1} \cdot$ Robert Berent $^{3}$ \\ ${ }^{1}$ Institut zur Diagnose und Therapie von Atherosklerose und Fettstoffwechselstörungen, Institut Athos, \\ Wien, Österreich \\ ${ }^{2}$ Innere Medizin, Salzkammergut Klinikum Vöcklabruck, Vöcklabruck, Österreich \\ ${ }^{3}$ HerzReha Bad Ischl, Bad Ischl, Österreich
}

\section{Erratum zu:}

Kardiologe 2021

https://doi.org/10.1007/s12181-021-

00503-7

In dem ursprünglichen Artikel wurde eine Institutszugehörigkeit der Autorin Dr. Theresa Berent falsch angegeben. Die korrekten Institutszugehörigkeiten sind die folgenden:

- Institut zur Diagnose und Therapie von Atherosklerose und Fettstoffwechselstörungen, Institut Athos, Wien, Österreich

- Innere Medizin, Salzkammergut Klinikum Vöcklabruck, Österreich
Die Redaktion bittet, den Fehler zu entschuldigen, und um Beachtung der korrekten Version.

Der vollständige und korrigierte Artikel steht Ihnen auf www.springermedizin.de zur Verfügung. Bitte geben Sie dort den Beitragstitel in die Suche ein.

\section{Korrespondenzadresse}

\section{Dr. Theresa Berent}

Institut zur Diagnose und Therapie von Atherosklerose und Fettstoffwechselstörungen, Institut Athos

Nadlergasse 1/2/1, 1090 Wien, Österreich

theresa.berent@gmx.at 